\title{
La Prevención de Riesgos y la accidentabilidad Laboral. APORTACIONES DESDE la Psicología SOCIAL
}

\author{
Andrés Rodríguez Fernández \\ Antonio Delgado Padial \\ Universidad de Granada
}

El accidente y la enfermedad profesional, son, sin duda alguna, dos de los problemas más graves y de mayor relevancia que tiene planteados aún hoy el mundo del trabajo y la sociedad en general, ya que implica altos y variados costes humanos. Ahora bien, la accidentabilidad laboral o la pérdida de la salud no es un problema exclusivo del trabajador y del empresario, sino que ha de ser responsabilidad de la sociedad en su conjunto, pues tanto los accidentes como la salud en el trabajo son temas centrales desde una perspectiva política, económica y social. Desde el punto de vista científico, las líneas de investigación que se han seguido, quizás hayan estado excesivamente dirigidas hacia la restauración o intervención, aunque en la actualidad parecen desplazarse hacia el campo de la prevención.

Professional accidents and diseases are without any doubt two of the main problems in the labour world and the society in general, as it implies a great deal of human lives. Now, the workplace accident rate or the health loss is not simply a problem for employers or employees, it must also be the responsibility of the society as a whole, as both workplace accidents and health are political, economic and social topics. From a scientific point of view, the research approaches have mainly been focussed towards restoration and intervention. However, at present research is developing towards prevention.

Palabras clave: Prevención, condiciones de trabajo, accidentabilidad, psicosociologia

Key words: Prevention, work conditions, accident rate, psychosociology

\section{INTRODUCCION}

Un tema de vital importancia en el mundo del trabajo ha sido y sigue siendo la prevención de rịesgos y la accidentabilidad laboral; sin embargo, esta relevancia 
intrínseca del fenómeno contrasta con la despreocupación social existente sobre la prevención y seguridad en el trabajo, que sólo alcanzará un cierto grado de sensibilización en momentos concretos de grandes catástrofes o cuando aparecen las estadísticas. En esos momentos establecemos hasta un día cada año para dedicar a la seguridad y a la salud en el trabajo ( 28 de Abril), según la Orden Ministerial de 30 de Marzo, y creamos una Fundación para que vele por la seguridad y salud de los trabajadores, y elaboramos un Plan de Acción sobre la Siniestralidad Laboral, y sellamos Protocolos-Declaraciones, y realizamos Cumbres y Conferencias sectoriales y generales, y organizamos Congresos y Simposia... Con toda esta parafernalia parecería que realmente nos interesa el tema de la seguridad y de la salud a todos, pero sólo es una mera ficción. Vivimos en una sociedad de apariencias y ficciones. Sólo importa la ficción, pero es tan desolador cuando dejamos de creer en las ficciones y nos enfrentamos a la cruda realidad. A pesar de todo, intentaremos crear una ficción, creérnosla y hacer que se la crea usted, estimado lector, cuando lea estas páginas.

$\mathrm{Si}$, como hemos dicho, las muertes por accidente de trabajo no tienen apenas repercusión social, a no ser que se enmarquen en desastres de grandes dimensiones, menos aún tendrán las enfermedades profesionales que, en general, se presentan de forma gradual y progresiva. Es paradójico y lamentable que a finales del siglo XX y con los avances técnicos que se han logrado en los sistemas de producción exista aún un número tan alto de trabajos que constituyan una amenaza para la salud e integridad física de los trabajadores, debido a los múltiples productos y sustancias tóxicas que han de utilizar en el proceso de transformación y. a las condiciones de trabajo tan deplorables en que aún se realizan múltiples actividades en diversos sectores. Además, lo grave es que la principal causa de la siniestralidad no es otra que el incumplimiento generalizado de las normas preventivas por parte de las empresas y de los trabajadores, y detrás de ese incumplimiento lo que subyace es una falta de cultura orientada a la prevención que, como es lógico pensar, no se transforma sólo con gestos y declaraciones.

\section{CONDICIONES DE TRABAjO}

Las medidas de ingeniería tanto en la protección de las máquinas como en la mejora de las condiciones de trabajo han sido decisivas, incluso en la actualidad se las reconoce como un instrumento eficaz y útil a la hora de reducir los indices de accidentabilidad laboral. No obstante, es claro que son insuficientes por sí solas para la consecución de tal objetivo.

Técnicos, trabajadores y empresarios coinciden en subrayar la gran importancia de la conducta del trabajador en la génesis de los accidentes laborales 
(Reason, 1990). Ello no implica, sin embargo, que toda la responsabilidad deba recaer sobre el operador humano con el consiguiente desplazamiento del énfasis técnico al psicológico. Por tanto, aunque pueda ser cierto que, en última instancia, todos los accidentes de trabajo puedan imputarse, directa o indirectamente a errores humanos, ya que estarán relacionados, bien con el diseño, construcción, equipamiento, dirección, con la supervisión en la organización o con la utilización de herramientas, máquinas, aparatos, vehículos o instalaciones; sin embargo, ello no debe hacernos olvidar la importancia que tiene el puesto de trabajo, los materiales y equipos con los que se trabaje y la estructura global de la organización (Reason, 1995).

Cuando en el trabajo se mantienen posturas incorrectas y se realizan movimientos inadecuados se provoca cansancio innecesario, bajo rendimiento y, frecuentemente, se producen accidentes o, cuando menos, dolencias óseo-musculares que suelen ser una causa importante de baja laboral y, además, éstas afectan no sólo a actividades consideradas duras (construcción), sino también a otras aparentemente más relajadas (banca, oficinistas).

Se ha comprobado que se puede lograr hacer una misma actividad con un menor esfuerzo. Las herramientas y el mobiliario condicionan muchas veces la forma de actuar, de ahí la importancia de aprender a adoptar las posturas correctas. Si pensamos en la postura y en los movimientos que empleamos en el trabajo y entrenamos a los trabajadores a desarrollar los correctos, podremos lograr unas condiciones de trabajo más saludables. Es una pequeña pérdida de tiempo que, sin duda, se compensaría por los beneficios que obtendríamos, sobre todo, en calidad de vida laboral.

En este sentido, las características que definen la mayor parte de los puestos de trabajo en la actualidad en el contexto europeo, según los resultados de la II Encuesta Europea sobre Condiciones de Trabajo realizada en 1996 por la Fundación Europea para la Mejora de las Condiciones de Trabajo, podríamos resumirlas en las siguientes: trabajo parcializado, de ciclo productivo excesivamente corto, repetitivo, de ritmo de producción continuo, generalmente en cadena, exigen un alto nivel de atención hacia la tarea, ausencia de autonomía...

Así, los cambios que se han producido en el mercado de trabajo, orientados hacia una mayor flexibilidad, han generado un aumento de los contratos temporales en la mayoría de los países de la Unión Europea, pues en 1996, aunque la media se situaba alrededor del $15 \%$, los porcentajes variaban de forma considerable entre los diferentes Estados miembros de la UE, pues mientras sólo afectaba este tipo de contratos a un $9 \%$ en Austria y Luxemburgo, alcanzaba al $40 \%$ en España.

A través de las investigaciones realizadas en varios países europeos se demuestra la existencia de mayor riesgo de siniestralidad y problemas de salud relacionados con el trabajo entre los asalariados con contratos temporales. 
Concretamente, un análisis de la Encuesta Europea sobre las Condiciones de Trabajo permite apreciar'que los trabajadores temporales sufren peores condiciones de trabajo. Así, un $38 \%$ está expuesto a ruido elevado, frente a un $29 \%$ de trabajadores fijos; un $57 \%$ adopta posturas dolorosas, frente a un $\mathbf{4 2} \%$ de los fijos; un $66 \%$ realiza movimientos repetitivos y solamente un $55 \%$ de los trabajadores. permanentes los efectúan.

En este sentido, se constata también que los trabajadores con contratos de duración determinada hablan menos de sus problemas laborales con sus compañeros, representantes y superiores, reciben menos información y se sienten víctimas de la violencia psicológica en mayor medida que los trabajadores con contratos indefinidos. Por tanto, a partir de estos datos se evidencia que los diferentes tipos de contratación tienen un efecto sobre la salud, independientemente de las condiciones de trabajo.

Las condiciones de trabajo de los empleos ocupados por los trabajadores temporales explican en gran medida, aunque no totalmente, los resultados mencionados anteriormente (la mayoría de los contratos de duración determinada se concentran en los sectores con altas tasas de trabajo estacional como la agricultura, la hostelería y la construcción). En conclusión, los trabajadores con contratos temporales están expuestos a peores condiciones de trabajo, se concentran en determinados grupos del mercado de trabajo y sufren con mayor frecuencia problemas de salud relacionados con el trabajo.

Algo similar ocurre con el trabajo continuo de noche o por turnos. En ambos casos esta demostrado que se producen alteraciones del equilibrio biológico y social de los trabajadores, dando lugar a problemas fisiológicos, psicológicos y sociales. Las consecuencias de estas características son, a medio y largo plazo, de una inusitada gravedad para la salud y el bienestar del trabajador, que podemos observar en fenómenos tales como trastornos gastrointestinales, trastornos nerviosos, pérdida de apetito, problemas de fatiga, que no logra hacer desaparecer durante los días de descanso y esa continuidad lleva a un claro deterioro en la calidad humana del trabajo, a una erosión de las relaciones interpersonales y, fuera de la empresa, al empobrecimiento de la vida familiar y comunitaria. Pero, además, èsas consecuencias no afectan sólo al trabajador, también tienen su impacto en la empresa a través de su actividad laboral.

Estas agresiones psicológicas y sociales se afianzan aún más por el hecho dee que la vida del ser humano en el trabajo monopoliza de forma abusiva su vida familiar y social. La jornada laboral, por su duración, absorbe una gran parte del tiempo útil, no deja tiempo libre para hacer más estrecha, más enriquuecedora y creativa la sociabilidad familiar y comunitaria. Estas contaminaciones provocadas, directa o indirectamente, por la actividad laboral, invaden tanto los 
ambientes internos como externos del trabajo, determinando una patología psicológica y social de la que el accidente no es más que otra desgraciada consecuencia.

Contrariamente a lo que cabría esperar, las condiciones de trabajo han empeorado en términos generales durante las últimas décadas, como ya a mitad de los años setenta anunciara Braverman (1975). El progreso técnico, que reduce los riesgos naturales, puede incrementar, en cambio, los que resultan del uso de la propia técnica.

Así, los países industrializados han desarrollado una intensa labor para mejorar dichas condiciones, si bien los sondeos de encuesta realizados entre expertos de estos países indican que la situación actual aún está muy lejos de ser óptima. Consciente de ello, la OIT ya adoptó por unanimidad llevar a cabo un programa tendente a la mejora de las condiciones de trabajo de los países miembros, denominado PIACT. De forma similar, aunque con igual éxito, se plantean el problema en la Unión.Europea a través de sus conocidos estudios y Directivas.

En España las condiciones de trabajo se sitúan muy por debajo de las que encontramos en los países de la Comunidad Europea, entre otras razones, por el menor grado de desarrollo tecnológico y organizativo, así como por el menor nivel de participación de la clase trabajadora en las tareas de planificación y diseño en los distintos procesos de la actividad laboral. En este sentido, algunos sindicatos subrayan que la "experiencia obrera" ha sido sistemáticamente rechazada o eludida por parte de los empresarios, lo que ha imposibilitado integrar el aprendizaje acumulado de cada trabajador y del grupo obrero en la planificación general de la actividad laboral. En consecuencia, habrá que tender a una superación de los modelos culturales imperantes que representan al obrero como un ser profundamente pasivo e incapaz de aprovechar una experiencia acumulada.

Independientemente de las características que definen la mayor parte de los puestos de trabajo en la actualidad y de la ausencia de participación de los trabajadores en la planificación de la actividad laboral, cuyas consecuencias ya hemos descrito aunque sea de forma breve, existen otros importantes estresores como las características de la organización, la presión de rol y las demandas de la tarea que, asociados a específicas condiciones de trabajo, rápidamente elicitan respuestas de estrés. Sin embargo, el grado de estrés estará moderado por las diferencias individuales.

Entre los estresores relativos a las condiciones físicas que más afectan a los niveles de desempeño y a la salud de los trabajadores destaca el ruido (Topf, 1985), ampliamente estudiado por los psicólogos, quienes pretenden identificar, en primer lugar, aquellas tareas cuyo desempeño está fuertemente determinado por el ruido $y$, en segundo lugar, el grado de sensibilidad de la gente a éste; ambas líneas de 
investigación pueden conducir a mejorar el ajuste entre trabajador y lugar de trabajo, impidiendo con ello que múltiples puestos sigan actuando como fuentes claras de pérdida de audición. Hemos de pensar que el ruido sigue siendo un aspecto del entorno físico que afecta a un elevado número de trabajadores. (La Benz, Cohen y Pearson, 1997).

Un segundo aspecto a considerar relativo a las condiciones físicas de trabajo es la temperatura, o mejor, el desajuste producido entre temperatura, grado de humedad y calor del aire (Vickroy, Shaw y Fisher, 1982). Lógicamente, cuando ambos estresores van juntos se producirá un efecto aditivo y, en consecuencia, un mayor malestar en los trabajadores sometidos a esas condiciones de trabajo.

La fatiga es otra de las variables que afectan tanto al desempeño como a la salud de los trabajadores. Existen distintas clases de fatiga: muscular, mental, emocional y de habilidades, si bien todas ellas están constituidas por componentes fisiológicos y psicológicos. Los cuatro tipos de fatiga están asociados con cambios neuroquímicos en la sangre que determinan la capacidad de respuesta de los sujetos ante una situación dada, de ahí su importancia en la génesis de los accidentes laborales. No obstante, hemos de tener en cuenta que la gente difiere en cuanto a la susceptibilidad a la fatiga y, a su vez, los puestos de trabajo difieren también en cuanto a la fatiga inducida o generada. En suma, la fatiga ha de considerarse como el producto de un desajuste entre nuestras habilidades y las demandas del entorno.

La Fundación Europea para la Mejora de las Condiciones de Vida y de Trabajo, ha resumido los resultados de diversas encuestas sobre las condiciones de trabajo en Europa llevadas a cabo por la propia Fundación y por los Estados miembros desde el año 1991 a 1996, cuyo resumen se recoge en una publicación reciente de la propia Fundación titulada: Les contraintes de temps et I'autonomie au travail dans l'Union, de la que extraemos algunas conclusiones.

La intensidad del trabajo está aumentando en todos los Estados miembros, pues; en general, las personas trabajan más rápidamente y han de cumplir plazes más ajustados. Y aunque la autonomía en el trabajo está también aumentando; este incremento no es suficiente para compensar la mayor intensidad del trabajø, que lleva aparejada una gran tensión y tiende a provocar estrés laboral. Este tipo de trabajo lo desempeñan, sobre todo, y por este orden, los trabajadores manuales especializados, los trabajadores de los sectores del transporte, la restauración y la metalurgia, así como los trabajadores jóvenes. Concretamente, el $28 \%$ de los trabajadóres que realizan trabajos de mucha tensión (intensidad elevada y escasa autonomía) sufren estrés, y el $20 \%$ burn out.

De hecho, más de la mitad de los trabajadores que están sometidos a grandes tensiones en el trabajo dicen que su salud o su seguridad se encuentran en peli- 
gro. En cambio, los trabajadores que gozan de mucha autonomía en su trabajo y han de hacer frente a elevadas exigencias, dicen padecer problemas de salud en porcentajes significativamente más bajos. Así mismo, los trabajos de dirección en Europa están sometidos a grandes presiones de tiempo, pero contienen suficiente grado de autonomía como para que esas exigencias se puedan controlar.

El transporte y la industria son los sectores que parecen generar mayor grado de tensión, mientras que los trabajos que se desarrollan en las áreas de administración y servicios son los que predominan en la categoría de bajo grado de tensión.

Todos los estresores a los que nos hemos referido actúan en la vida laboral como agentes potenciales de disfuncionamiento o de accidentes (Williams, Calhonn y Ackoff, 1982). Por tanto, en la medida que seamos efectivos en su reducción o en su control, habremos eliminado posibles fuentes de riesgo e incrementado, consecuentemente, los niveles de seguridad en el contexto laboral. En este sentido, algunas empresas están adoptando una postura activa al respecto, esforzándose para que sus empleados trabajen a gusto. La revista FORTUNE ha constatado mediante dos "rankings" publicados en 1998 que existe una gran coincidencia entre las empresas mejor dirigidas y aquellas en que los empleados están mas satisfechos con sus condiciones de trabajo. Por tanto, ya que esforzarse por mejorar las condiciones laborales es rentable, tanto más lo será invertir en la prevención de riesgos laborales, puesto que es la primera e indispensable condición para sentirse cómodo en el trabajo.

RELEVANCLA DEL FENÓMENO:

Este año superaremos con toda probabilidad el millar de muertes en el trabajo y superaremos también las ochocientas mil bajas laborales y en este falaz sentido de superación se incrementarán, asimismo, los costes derivados de los accidentes laborales que ya el año pasado se situaron en torno a los dos billones de pesetas.

El accidente y la enfermedad profesional, derivados de las condiciones de trabajo en que se desarrolla la actividad laboral, son, sin duda alguna, dos de los problemas más graves y de mayor relevancia que tiene planteados aún hoy el mundo del trabajo y la sociedad en general.

Las catástrofes de Italia, México, la India o la Unión Soviética son claros exponentes de los riesgos potenciales que entraña la actividad laboral en ciertos sectores que, aunque de forma indirecta y más difusa, afectarán notablemente a la calidad de vida general, sometida a unos niveles excesivos de toxicidad. Incluso en nuestro país, ciudades como Huelva, Tarragona, Avilés o Puertollano son ejemplos 
claros de las agresiones sistemáticas a las que estamos sometidos todos, trabajadores y no trabajadores. Según la O.M.S, la mayor parte de las enfermedades cancerígenas (80\%) tienen un origen ambiental, de las que la mitad aproximadamente se deben al medio ambiente laboral.

Pero, con independencia de estos sucesos concretos, ciertamente impactantes, existe una realidad laboral cotidiana, que implica altos y variados costes humanos. El accidente -con lesiones- es el indicador más claro y visible de tal realidad, si bien esos costes pueden traducirse, asimismo, en enfermedades profesionales, fatiga, envejecimiento prematuro y trastornos derivados de la insatisfacción en el trabajo. Todos estos aspectos disfuncionales son, en sentido estricto, accidentes laborales, ya que implican un desajuste en el sistema, concretamente en el componente humano.

Estamos asistiendo, pues, durante las últimas décadas en nuestro país a una patología más difusa: las transformaciones generadas en la organización del trabajo, la progresiva sofisticación alcanzada por la tecnología con la que debe operar el trabajador, sin estar aún adaptado a ella, el persistente riesgo potencial de la energía nuclear tan ampliamente generalizada, confirman la gravedad real y potencial del fenómeno.

Por tanto, se observa un primer nivel constituido por esos sucesos específicos, espontáneos y trágicos: los accidentes con lesión; un segundo nivel relativo al sistemático y progresivo deterioro de las condiciones de trabajo que llevarán a la enfermedad profesional o al envejecimiento prematuro, entre otras consecuencias $y$, finalmente, un tercer nivel constituido por el deterioro del nivel de calidad de vida general, que afecta a la comunidad en su conjunto.

Desde esta perspectiva, la salud en el trabajo trasciende el estricto marco de la empresa y, obviamente, no puede considerarse desde la percepción del individuo o de la propia organización; antes al contrario, se debe contemplar como un hecho social. En consecuencia, la accidentabilidad laboral o la pérdida de la salud no es un problema exclusivo del trabajador y del empresario, sino que ha de ser responsabilidad de la sociedad en su conjunto, pues tanto los accidentes como la salud en el trabajo son temas centrales hoy, desde una perspectiva política, económica y social. Desde el punto de vista científico, la relevancia es obvia, púres desde principios de siglo se están investigando distintos aspectos de la accidentâbilidad a partir de variados marcos teóricos y metodológicos. No obstante, las linèas de investigación que se han seguido, quizás hayan estado excesivamente dirigidas hacia la restauración o intervención, aunque en la actualidad parecen desplàzarse hacia el campo de la prevención. 


\section{DEFINICIÓN DE ACCIDENTE}

Haciendo un breve análisis acerca de las múltiples definiciones existentes sobre accidentabilidad laboral se observa un bajo grado de acuerdo entre los estudiosos que trabajan sobre el tema, lo que entraña ciertos problemas para el desarrollo de la investigación. Podrían sugerirse cuatro perspectivas, al menos, en las que enmarcar la mayor parte de las definiciones planteadas: médica, legalista, economicista y ergonómica, según enfaticen las consecuencias físicas y traumáticas localizadas en la víctima, los aspectos legales y normativos en relación con la responsabilidad del accidente, los aspectos económicos derivados de éste o el proceso mismo del accidente concebido en sus dimensiones más básicas.

Independientemente de cuál sea el punto de partida, la mayoría de los autores coinciden en resaltar una característica del accidente: considerar que éste implica una interrupción del funcionamiento normal y adecuado del sistema. Esta característica coincide con la definición que se viene dando desde el campo de la ergonomía, fundamentalmente, por Leplat y Cuny (1979). Por tanto, la definición que proponemos tan sólo añade algún matiz a la ofrecida por estos autores:

\section{"El acctdente es un disfuncionamiento del sistema, que puede afectar al operador bumano (accidente con lesiones o mortal) o no (accidente en blanco)".}

MODOS DE CLASIFICACIÓN Y FUENTES DE DESAJUSTE EN LA PRODUCCIÓN DE ACCIDENTES LABORALES

Un modo eficaz que se viene utilizando para arrojar luz sobre las causas reales de los accidentes de trabajo es su clasificación. En este sentido, unos investigadores han desarrollado métodos para clasificar los accidentes según sus causas, que básicamente podríamos reducir a los sistemas de clasificación simple y sistemas de clasificación múltiple, dependiendo de que la causa del accidente se considere como única o múltiple. Otros, por el contrario, intentan clasificar los accidentes, no de acuerdo con sus causas, sino sobre la base del agente responsable. Sin embargo, sean unos u otros, los métodos que se sigan no resolverán el problema, ya que en general las opiniones sobre la verdadera causa de un accidente suelen ser muy heterogéneas y las causas, además, pueden ser múltiples.

Por ello, se han intentado elaborar sistemas de clasificación más complejos, según los cuales cada accidente se registraría a partir de distintos criterios: agente material, tipo de trabajo, deficiencia del agente material, actos imprudentes, factores psicológicos y sociales y condiciones físicas de trabajo. Otros sistemas de cla- 
sificación, aún siendo múltiples, se centran en aspectos distintos: tipo y caracterís:ticas de la lesión, parte dèl cuerpo afectada, origen de la lesión, tipo de accidente, condición peligrosa que permitió el accidente, agente material del accidente y acto imprudente.

Ante esta falta de homogeneidad, la OIT adoptó un sistema de clasificación basado en los siguientes criterios: forma de accidente, agente material, naturaleza de la lesión y ubicación de la lesión. De todos modos, sea cual sea el sistema de clasificación elegido, parece que la mayoría de los accidentes no ocurren de forma sistemática ni con las máquinas de mayor riesgo, ni con las sustancias más peligrosas, ni entre los trabajadores más inexpertos. De otro lado, en los distintos sistemas de clasificación que hemos comentado, se asume que el accidente ocurre como consecuencia de un conjunto de factores y sólo muy raras veces podrá deberse a un solo factor.

Por lo que se refiere a las fuentes de desajuste, esbozaremos dos sistemas de categorías de estudio, que podrán ayudarnos a analizar el accidente de trabajo de una forma más sistemática y totalizadora. Así pues, y desde el enfoque ergonómico, Leplat y Cuny (1979) delimitan cuatro niveles organizativos donde se originarían esos posibles desajustes:

- Nivel de organización, concebida como un todo.

- Nivel de servicios.

- Nivel de grupo de trabajo.

- Nivel de puesto de trabajo.

En el primero de esos niveles se produciría el accidente, bien por una falta de ajuste del nivel administrativo a las unidades funcionales, por interferencias en la comunicación, o bien por una insuficiente coordinación de las actividades.

En el segundo nivel, los accidentes pueden producirse a causa de una deficiente distribución de las tareas, insuficiencia de las normas que se dan para ejecutar el trabajo o a causa de inestabilidad funcional e inestabilidad social en el sistema.

En el tercer nivel, las alteraciones podrían producirse, bien por interferencias y bloqueos en la circulación de la información o por defectos en la cohesión dèl grupo.

Finalmente, en el cuarto nivel, relativo al puesto de trabajo, las fuentes de desajuste pueden deberse tante a los materiales utilizados, organización del trabajo; condiciones ambientales, como a las características del trabajador:

Por su parte, De Reamer (1980), desde una perspectiva más concreta, al cuèstionarse las causas de los accidentes, comienza por distinguir los que según él son los dos factores más importantes en la producción de éstos: 
- Las condiciones inseguras, que incluyen: errores en el diseño de los equipos, errores en la ubicación o ensamblaje de los equipos, situaciones físicas peligrosas y utilización de quipos en mal estado.

- Los actos inseguros, que cubren: el uso inapropiado del equipo, conductas inapropiadas y bromas.

A partir de estas dos causas genéricas básicas, presenta algunos supuestos sobre la génesis del accidente que, de un modo breve, podríamos resumir como sigue:

a) El comportamiento del supervisor y la condición física y mental del trabajador son las principales fuentes de los actos y de las condiciones inseguras.

b) No todos los actos y condiciones inseguras son generadoras de accidentes, sino que todos los accidentes resultan de actos y condiciones inseguras, generalmente en combinación.

c) Todos los accidentes de trabajo producen demoras y causan daños o deterioros materiales.

d) Algunos accidentes causan daños personales, si bien sólo en una pequeña proporción tales daños llegan a ser mortales.

En relación con los actos inseguros, Bird (1994) sugiere que la gente tiene unas necesidades de seguridad, pero a la vez desea cubrir otras que entran en conflicto con las primeras. Así, el deseo de ahorrar tiempo y esfuerzo, ganar en bienestar, atraerse la atención de los compañeros, actuar de forma independiente, ganarse la aceptación de los demás, son necesidades que los trabajadores intentarán cubrir aunque entre, a veces, en conflicto con la necesidad de seguridad. En consecuencia, los psicólogos habrán de ofrecer estrategias de cambio de conducta a fin de reducir al máximo la frecuencia de esas conductas, puesto que se parte del supuesto de que toda conducta insegura es aprendida $y$, por tanto, modificable. En este sentido, el psicólogo puede sugerir diversos métodos para promover la seguridad en el trabajo: reglamentación de normas, estandarización de dispositivos de protección, inspección, desarrollo de investigaciones técnicas, médicas, psicosociológicas y estadísticas, educación sobre normas de seguridad, formación profesional, persuasión y propaganda, configurando así una conciencia social más orientada hacia la seguridad.

Cada día la difusión del desarrollo tecnológico es mayor, la variedad de productos químicos utilizados, creciente, y los riesgos derivados de las radiaciones y de la energía atómica cada vez más generalizados. Todas estas situaciones de riesgo real que acompañan al progreso no deben entenderse, sin embargo, como la factura fatídica que el ser humano tiene que pagar si quiere conseguir altos nive- 
les de bienestar, pues el largo programa que culminó con la llegada del hombre a la luna y su actual permañencia en el espacio ha implicado enormes avances en el campo tecnológico y, en cambio, los accidentes producidos han sido mínimos. Por tanto, el progreso tecnológico no tiene necesariamente por qué implicar un incremento en los niveles de riesgo para el individuo, sino todo lo contrario.

\section{LA INVESTIGACIÓN DEL ACCIDENTE}

Hemos de partir del supuesto de que todo accidente, aún el más insignificante, debe ser comunicado e investigado. El objetivo de la investigación de un accidente es encontrar las causas que lo han producido y adoptar las medidas oportunas para que no pueda suceder de nuevo. Para ello se procura averiguar las causas del accidente, determinar el cambio o error que se produjo en el sistema, comunicarlo a trabajadores y supervisores y delimitar los hechos y opiniones.

En general los investigadores suelen utilizar la técnica de las seis preguntas: ¿Quién resultó lesionado? ¿Qué ocurrió y qué factores contribuyeron a ello? ¿Cuándo sucedió? ¿Dónde sucedió? ¿Por qué ocurrió? ¿Cómo puede impedirse que se repita? La investigación de un accidente ha de hacerse siempre en el mismo lugar donde ocurrió y, en la medida en que se pueda, recreando el escenario de la forma más parecida posible a la que existiera en el momento del suceso.

\section{MARCO TEÓRICO}

En el estudio sobre accidentabilidad laboral no existe una perspectiva unitaria, sino más bien una gran variedad de enfoques que tan sólo tienen en común su preocupación por explicar las causas del accidente. Veamos a continuación algunos de los más representativos.

\section{Enfoque de las diferencias individuales:}

Hasta hace poco tiempo se mantenía como axioma el supuesto de que los accidentes laborales eran causados, fundamentalmente, por errores humanos. Por ello no debe sorprender que gran parte de la investigación se haya orientado hacia el establecimiento de relaciones entre una amplia gama de, capacidades y accidentes, en un esfuerzo por identificar el perfil psicológico ideal de un trabajador seguro; sin embargo, los resultados de tales estudios ofrecen correlaciones muy bajas. Tales hallazgos indican que el desempeño de esas capacidades puede explicar solamente una parte mínima de la varianza total o, dicho de otra forma, el accidente casi 
nunca se debe a una sola causa y las posibles fuentes de desajuste, como ya se ha visto, pueden ser múltiples.

Los primeros estudios que se desarrollan sobre el tema se enmarcan dentro del enfoque diferencialista. Así, el estudio científico de los accidentes laborales se inicia con la "teoría de la propensión", que se fundamenta en el hecho observado de que en la mayoría de los grupos de trabajo estudiados, una minoría es responsable de la mayor parte de los accidentes.

El concepto de propensión deriva directamente de los estudios estadísticos, si bien tales estadísticas deben ser muy completas para que puedan ser útiles y, de otro lado, es muy difícil seleccionar objetivamente, de entre todos los posibles factores, aquellos específicos a los que se pueda atribuir el accidente. En general, los estudios realizados desde el marco de esta teoría han resultado decepcionantes, puesto que, en realidad, las personas que han tenido varios accidentes no presentan en un examen psicológico perfiles significativamente distintos de aquellos otros que no han sufrido ninguno (Siguán, 1981).

Los estudios estadísticos realizados sobre la incidencia del factor humano en el origen de los accidentes muestran la importancia de variables tales como personalidad, inteligencia, antigüedad en el puesto, nivel de cualificación, edad o sexo; sin embargo, interpretar esas informaciones con exactitud no es fácil por el solapamiento que se dan entre algunas de ellas (edad, antigüedad, cualificación). Pero, además, estas variables interactúan con muchas otras relativas al medio ambiente: hora del día en que ocurren los accidentes o día de la semana, lo cual complica aún más las posibles interpretaciones que puedan darse.

No obstante, las estadísticas sobre accidentes son indispensables para elaborar programas adecuados de prevención. Así, podemos saber a través de las estadísticas qué tipo de trabajadores son los más afectados, qué máquinas o equipos los provocan, en qué horas y lugares se producen o qué conductas realizó el individuo inmediatamente antes del accidente, si bien sólo pueden reflejar los datos que se hayan notificado mediante el parte del accidente, que sólo se hace si implica baja. Por tanto, las estadísticas no pueden recoger todos aquellos accidentes que, afortunadamente, no han producido lesiones graves, pero que son tan importantes - más que éstos con respecto a la prevención.

\section{Enfoque cognitivo}

En el campo de la accidentabilidad laboral es realmente importante el estudio de los procesos cognitivos, pues en todas las tareas, sean del tipo que sean, se hayan implicados, si bien serán en las tareas cognitivamente complejas donde tengan una mayor incidencia (Sperandio, 1980). 
En la actualidad, parece imprescindible contar en la investigación sobre accidentes de trabajo al menos̄ con tres procesos cognitivos básicos: procesamiento de información, solución de problemas y toma de decisiones, a fin de poder explicar el comportamiento humano implicado (Rasmussen, Brehmer y Leplat, 1991). Así, el individuo, al desarrollar su actividad ha de coordinarla necesariamente con las actividades de otros para lograr unos objetivos determinados. Este proceso implica, como es lógico, un continuo procesamiento de información, adoptar constantes decisiones y resolver un gran número de problemas hasta alcanzar esos objetivos de una forma eficaz y satisfactoria. Cualquier error que pudiera producirse en cualesquiera de estos procesos afectaría al sistema y, consecuentemente, cabría un riesgo de alteración y de accidente (Leplat y De Terssac, 1990).

Sin embargo, un análisis de éste, centrado en la conducta del individuo, no será adecuado, ya que se da en un contexto en el que tal conducta interacciona permanentemente con la tecnología, estructura organizativa y relaciones de poder inherente a ésta. Por tanto, la conducta individual ha de contemplarse desde el marco de la organización, concebida en la actualidad como un sistema complejo de procesamiento de información o como una entidad sociocognitiva. El individuo, de otra parte, necesita establecer unos niveles de referencia en torno a la seguridad para poder reajustar en todo momento sus pautas de conducta, y tales niveles de referencia, obviamente, se dan en la organización como un sistema social.

Bajo este enfoque, por tanto, la investigación sobre accidentabilidad laboral se hace enormemente compleja, debido al amplio abanico de variables y niveles que se han de considerar, así como a las dificultades que entraña su operacionalización en el ámbito de las organizaciones. De otra parte, un elevado número de conductas desarrolladas por los operadores humanos en su actividad laboral se realizan de un modo rutinario y mecánico, difíciles, por tanto, de analizar desde el marco de la racionalidad que inspira el enfoque cognitivo. Quizá, por ello, y porque todavía es reciente el resurgimiento de este enfoque, aún teniendo ya incidencia en el campo de las organizaciones (Peiró, 1984), no se há generalizado a otras áreas más específicas como es, en nuestro caso, el fenómeno de la accidentabilidad laboral.

\section{Enfoque psicosocial:}

La perspectiva psicosocial ha tenido una fuerte incidencia en la conșolidación y desarrollo de la Psicología de las Organizaciones en general; por tanto, cualquier análisis que se realice en torno a los desajustes producidos en éstas ha de tener en cuenta tal enfoque (Dodier, 1995). 
Concretamente, el papel que juegan los grupos en la producción de accidentes ha sido considerado por la literatura de muy diverso modo, en cuanto a su importancia. Sin embargo, parece lógico pensar que el grupo, más aún si está bien cohesionado, será un elemento decisivo en relación con la seguridad, tanto para el comportamiento de los miembros como para el de otros grupos y el de la organización en su conjunto (Kouabenan, 1999).

Asimismo, los aspectos relativos a la integración de los individuos y de los grupos con las actividades y fines de la organización es otra faceta importante a considerar desde el punto de vista de la seguridad. No hay que olvidar que la organización ha de contemplarse también en su dimensión social, lo que la hace ser un verdadero y eficaz agente de socialización y, desde esta perspectiva, puede establecer normas y pautas de comportamiento orientadas a conseguir un mayor grado de seguridad y de calidad de vida en el trabajo (Carlsson, 1980).

Los estilos de supervisión empleados por la dirección y los mandos ha sido otra área ampliamente estudiada desde la perspectiva de la seguridad (Román et al., 1973).

Los estudios que se han desarrollado acerca de las actitudes de los trabajadores hacia el trabajo han sido prolijos. Destacan los trabajos que se orientan a delimitar la importancia real de las actitudes seguras de los superiores al actuar como modelos de conducta para los subordinados (Vandenput, 1970). También se están desarrollando programas de modificación y cambio de actitudes orientados a la consecución de comportamientos más seguros (López Mena, 1978).

Por último, el estudio de los canales y de las barreras de comunicación en los distintos niveles de las organizaciones con respecto a la prevención de accidentes ha sido una de las líneas de investigación a las que se han venido dedicando mayor atención en los ferrocarriles franceses, en la CECA y en otros sectores de la Unión Europea (Grosjean y Lacoste, 1999).

Las organizaciones, de otro lado, no pueden vivir aisladas, su propia dinámica cotidiana implica elementos ajenos a ella; los trabajadores están en permanente interacción con los distintos elementos del entorno, los cuales moldean sus actitudes en relación con la seguridad y con el propio trabajo. Así, pues, si entendemos la organización como un sistema abierto, es imprescindible analizar sus relaciones con el medio para conseguir una conducta organizativa coherente con el entorno cultural y geográfico en relación con la seguridad y la calidad de vida en el medio laboral.

En resumen, los procesos de integración y de socialización, conducta de grupo, actitudes, estilos de supervisión y canales de comunicación han sido los temas sobresalientes desde este enfoque en relación con los accidentes de trabajo. 


\section{Enfoque ergonómico:}

La Ergonomía puede concebirse como un enfoque orientado a la transformación de las condiciones de trabajo, implicando a los trabajadores afectados por dichas transformaciones, a los especialistas de diferentes disciplinas, y teniendo en cuenta el marco global en el que se desarrolla la actividad laboral (Laville, 1976; Holnagel, 1993).

La integración de la seguridad en los objetivos de la Ergonomía se establece a través de dos tipos de criterios. De una parte, criterios que traducen los componentes de inadaptación de las condiciones de trabajo (criterios de seguridad, de producción, psicofisiológicos y psicosociales). De otro lado, criterios de validación de la intervención ergonómica (criterios de optimización y de cargas de trabajo).

Bajo este enfoque, la conducta del operador y su estrategia de trabajo se sitúa y analiza en la propia organización, estudiándose la comunalidad de objetivos y las múltiples interacciones entre los diferentes elementos del conjunto. El centro de atención es la carga de trabajo a la que está sometido el operador durante su horario laboral.

Desde una perspectiva más global, la gran cantidad de factores que pueden afectar al sistema podríamos sintetizarlos, siguiendo a Moyen, Quinot y Heimfert (1980), en los siguientes:

- Carencia de algún útil, máquina o instalación con cuya presencia se debiera haber contado para la realización correcta de la tarea.

- Falta de fiabilidad de las herramientas, materiales, máquinas o instalaciones.

- Defectos en la concepción de las máquinas o de las tareas.

- Factores individuales que por desconocimiento o error pueden perpetuar el desarrollo previsto de la tarea.

- Omisión o ejecución parcial de algún elemento de la tarea.

- Situaciones peligrosas por su propia naturaleza, independientemente de la tarea.

Como puede observarse, se ha pasado de la causa única que pretendía explicar el accidente, a la multicausalidad implicada en la teoría de sistemas. Este cambio es de una importancia vital, pues implica una mayor capacidad de prevención al mismo tiempo que mejora y hace más duradero el nivel de seguridad en las organizaciones. Se ha pasado, por tanto, de unas estrategias de investigación basäđas en una imagen cartesiana del hombre, en donde el trabajador era concebido como una máquina, a un enfoque ergonómico y sistémico en el que el operador humáno se concibe como un elemento en interacción dinámica con el resto del sistema y del entorno social (Levenstein y Wooding, 1997). 


\section{ESTUDIO DE LAS VARIABLES RELEVANTES}

Los temas estudiados con mayor profusión en relación con los riesgos y con la accidentabilidad laboral podrian incluirse en cinco amplias categorías, bien entendido que el criterio utilizado en su elaboración ha sido el mero deseo de alcanzar un mayor grado de comprensión.

En la primera de ellas incluimos algunas variables que han planteado al menos un gran atractivo a los investigadores que se han ocupado del tema: inteligencia general, aptitudes específicas y personalidad, que, como podemos observar, son de carácter psicológico.

La segunda categoría integra distintas variables que durante las últimas décadas se les están prestando bastante atención tanto en el ámbito laboral como, sobre todo, en los estudios sobre circulación de tráfico: alcohol y otras drogas, estrés, fatiga y monotonía. Estas variables las consideramos de carácter psicopatológico en cuanto a sus efectos.

En la tercera categoría se integran aquellas otras variables de carácter más psicosocial, tales como

la comunicación, cooperación, motivación y satisfacción en el trabajo, estilos de supervisión y actitudes.

La cuarta categoría comprenderá aquellas variables más representativas relacionadas con el sistema organizativo en su dimensión más estructural: características de la tarea, carga de trabajo, adaptación al trabajo, condiciones ambientales, diseno de equipo e instrumentos, estado de la tecnología, rama de actividad, tamaño de la empresa y turnos de trabajo.

Finalmente, en la quinta categoría incluimos variables relacionadas con la maduración de los individuos, tales como la edad y la experiencia.

\section{VARIABLES PSICOLOGICAS:}

La realización de cualquier trabajo, es obvio, requiere ciertas aptitudes que permitan ejecutarlo con eficiencia. No obstante, su medición y la utilidad de ésta en la organización para poder predecir la accidentabilidad laboral no siempre es fácil, puesto que no tenemos seguridad de que ciertas aptitudes se presenten como aptitudes generales adecuadas para cualquier situación, sino que, por el contrario, presentan características distintas según las modalidades de cada tarea y, además, aunque la situación de examen reproduzca la tarea real, no es seguro que la atención del sujeto sea la misma en ambas situaciones. 
Además, y esto puede suponer un sesgo fundamental, el potencial aptitudinal que posea el individuo pùede cambiar al actualizarlo en una situación concreta, pues, independientemente de las interferencias que puedan suponer variables de tipo motivacional, de integración, comunicativas, de organización y tecnológicas a la hora de poner en acción dicho potencial, un sujeto puede abusar de una aptitud y con ello convertir ese alto potencial en algo negativo para sí mismo y para la organización; por el contrario, otro individuo con un bajo potencial en una aptitud determinada, podría sustituirla por otras y convertir así su potencial defectuoso en una ejecución eficaz y segura.

Inteligencia general: Teniendo en cuenta las observaciones anteriores, en la mayor parte de la literatura revisada se nos indica la ausencia de relación significativa entre la inteligencia de los trabajadores y sus respectivos índices de accidentes en la población general.

Aptitudes especificas: Entre las primeras materias investigadas en Psicología aplicada al mundo del trabajo, sobresalen las capacidades sensoriales, que aún hoy siguen ocupando un lugar de primer orden. Más específicamente, de todos los órganos sensoriales, aquél que con mayor intensidad se ha estudiado en relación con la accidentabilidad laboral ha sido, sin lugar a dudas, la visión (Mc Cormick, 1980).

Según se desprende de la investigación, las características visuales que tienen una gran importancia son múltiples, de entre ellas destacaríamos: agudeza próxima y remota, percepción de la distancia o de la profundidad, equilibrio muscular de los ojos y discriminación del color. Quizá por ello no se observa un consenso generalizado en cuanto a los resultados.

Psicomotricidad: Las variables de tipo psicomotor por las que se ha mostrado mayor interés en relación con la accidentabilidad, han sido aquéllas relacionadas con la rapidez y la coordinación (Alonso et al., 1979). A pesar de ello, considero pertinente aludir a las objeciones que hacen algunos autores a las pruebas de tipo motor al considerar que las aptitudes puestas en juego en los tests motores pueden cambiar con la práctica. Este cambio de naturaleza de muchas pruebas motoras, a consecuencia de la práctica, complica la determinación de la fiabilidad.

Personalidad: En general, los trabajos de investigación desarrolladös en el ärea de la personalidad han seguido dos pautas metodológicas significativamente dístintas: 
a) Comparación de grupos de poliaccidentados con grupos de sujetos no accidentados para averiguar las diferencias existentes entre ellos en una serie de rasgos de personalidad.

b) Estudios clínicos de sujetos poliaccidentados con el fin de encontrar rasgos comunes a todos ellos y poder así atribuir el hecho de que se accidenten con más frecuencia que los restantes trabajadores a dichos rasgos.

Las investigaciones desarrolladas desde la perspectiva psicoanalitica -las más cuantiosas - resultan difíciles de verificar $y$, posiblemente, la convergencia de resultados se deba más a la difusión de la teoría que a la realidad objetiva de los hechos (Helm, 1978).

Por último, cabe mencionar algunos estudios basados en la teoría de los biorritmos (Carvey y Nibler, 1977); Persinger, Cooke y Tanes 1978), que clurante las últimas décadas parecen tener cierto empuje, aunque tampoco han mostrado evidencia clara en cuanto a la relación entre los biorritmos de los sujetos y los accidentes laborales. Esta teoría parte del supuesto de que la conducta humana está determinada por tres ciclos llamados "ritmos biológicos" o "biorritmos": un ciclo físico de veintitrés días, un ciclo emocional de veintiocho días y un ciclo intelectual de treinta y tres días. El primero parece influir en las tareas físicas, el segundo en las tareas de alto contenido emocional y el tercero en aquellas ocupaciones que requieren razonamiento y juicio crítico.

Los ciclos de los biorritmos son curvas con una fase positiva, otra negativa y una tercera llamada fase cero o crítica, en la que la curva cruza el eje desde la fase positiva a la negativa o viceversa. Aunque los días negativos se consideran menos favorables que los positivos, son los días críticos -seis días al mes-los que se consideran más importantes, pues suelen ser los días en que, según los defensores de esta teoría, ocurre el mayor número de accidentes. De todos modos, los estudios realizados para intentar validar la teoría de los biorritmos como predictores de la accidentabilidad, sin embargo, han dado unos resultados altamente desalentadores (Floody, 1981).

Trataremos, por último, del exceso de confianza, una variable de personalidad sobre la que existe un mito generalizado, pues una de las creencias más arraigadas en la cultura preventiva, tanto de los empresarios como de los propios trabajadores, es que el exceso de confianza de estos últimos, es una las causas habituales de los accidentes de trabajo. Así, el exceso de confianza seria el principal impulsor de las aimprudencias" que preceden a la mayoría de los accidentes.

En general, los expertos coinciden en que la creencia aludida no es más que un mito, un mito doblemente útil. En primer lugar, útil como tranquilizante de las conciencias de todos aquellos que, por acción u omisión, contribuyeron a potenciar 
en exceso esa confianza. En segundo lugar, útil como pantalla tras la que disimular los fallos latentes què, en forma de errores de diseño de organización o de planificación, pudieran presentarse.

La importante novedad, es que los trabajadores están dejando rápidamente de creer en el mito del exceso de confianza, como demuestran las sucesivas Encuestas Nacionales de Condiciones de Trabajo que el INSHT ha realizado en 1987, 1993 y 1997.

En efecto, el porcentaje de trabajadores que creen que la primera causa de los accidentes de trabajo es el exceso de confianza ha descendido del $62 \%$ en 1987 al $41 \%$ en 1993 y al $28 \%$ en 1997 . A esta evolución le ha acompañado, lógicamente, el aumento de quienes creen que la principal causa de los accidentes radica en la peligrosidad inherente al propio trabajo: el porcentaje de quienes así opinan ha pasado del $21 \%$ en 1987 al $45 \%$ en 1997.

Un cambio significativo sobre el que es preciso reflexionar, porque si los trabajadores están abandonando su sentido de autoculpabilización, es de esperar que, poco a poco, vaya calando en nuestra sociedad una nueva creencia: que los accidentes de trabajo no son el fruto de la mala suerte ni de las imprudencias de los trabajadores, sino el resultado esperable de unas condiciones de trabajo inapropiadas.

VARIABLES DE CARÁCTER PSICOPATOLÓGICO:

Entre las variables de carácter psicopatológico que con mayor interés se han estudiado en el ámbito laboral por las repercusiones que tienen sobre la producción y sobre los accidentes de trabajo, cabrían citarse el alcoholismo y otros tipos de drogas. Los estudios realizados con respecto a estas variables han sido numerosos y bastante homogéneos en cuanto a los resultados (Levine, Greenbaum y Notkin, 1973; Hine, 1973; Benjamin, 1977). Parece existir un acuerdo generalizado por parte de los distintos autores en cuanto a los efectos negativos del alcohol y otras drogas para el desarrollo de comportamientos seguros.

Alcoholismo: : El fenómeno del alcoholismo sigue siendo un problema importante para las empresas españolas, a pesar de que el número de trabajadores que consumen alcohol en exceso durante los últimos diez años ha descendido. Sin embargo, sigue siendo un problema grave, pues estudios recientes, como el realizado por investigadores de la Universidad de Murcia (publicado en la revista Arch. mal. prof. 1996, 57, $\mathrm{n}^{\circ}$ 5) muestran que las principales consecuencias de un consumo excesivo de alcohol en el trabajo son: el incremento de la morbilidad y el absentismo, el aumento del número de accidentes de trabajo y la disminución del rendimiento laboral. 
la Prevención de Riesgos y la accidentabilidad laboral. Aportaciones desde la Psicolocía Social 91

Algunas empresas españolas han realizado campañas y actuaciones para combatir el uso abusivo del alcohol con buenos resultados. Asimismo, la Red Europea de Promoción de la Salud en el lugar de trabajo, integrada por 15 oficinas nacionales de diferentes países europeos, ha obtenido información acerca de las actividades de prevención y promoción de la salud que se han llevado a cabo en los últimos cinco años en las empresas españolas de más de 250 trabajadores. De las 927 empresas que contestaron el cuestionario sobre actividades preventivas en relación con el consumo de alcohol, un $40 \%$ de ellas realizaba de forma esporádica actuaciones para prevenir el alcoholismo, siendo la industria (con un 50\%) el sector que más se preocupaba por el tema. Las estrategias utilizadas consistían en charlas personalizadas, conferencias y entrega de documentación, prohibiéndose la venta de bebidas alcohólicas y desarrollando campañas de sensibilización a través de carteles y folletos. Los efectos de tales acciones se hicieron patentes a través de la mejora del clima laboral, la disminución del absentismo y de los accidentes laborales.

Adicción al trabajo: Otra de las variables de carácter psicopatológico que cada día adquiere mayor relevancia en las empresas es la adicción al trabajo. Por ello, cuando los psicólogos se refieren al trabajo como adicción se basan, fundamentalmente, en el gran impulso que muestran algunos individuos por entregarse a su trabajo de una forma preferente y con evidentes señales de malestar al verse obligados a apartarse de él, aunque sólo sea de forma temporal.

Esta adicción puede llegar a afectar a la salud cuando se da de forma desmedida y va acompañada de determinadas características. Los especialistas aconsejan adoptar medidas preventivas como:

- Ocupar el tiempo libre en una actividad que agrade.

- Establecer una escala de preferencias en la que la vida afectiva resulte reforzada.

- Recurrir a profesionales y realizar sesiones de terapia, si el caso es grave.

No obstante, el número de trabajadores afectados en nuestro país no alcanza las cifras de poblaciones laborales de otros países, como muestran algunos resultados de encuestas recientes (el $42,8 \%$ de los encuestados, esencialmente los hombres, estaría dispuesto a sacrificar un $10 \%$ de su salario e idéntico porcentaje de su jornada laboral, para ganar un $10 \%$ más de tiempo de ocio. Incluso, un $70 \%$ de los españoles admite, asimismo, que es tanto o más importante lo que hace con su tiempo libre que su propio trabajo. 
VARIABLES DE CARÁCTER PSICOSOCIAL:

Bajo este epígrafe trataremos de forma muy breve todas aquellas variables que configuran lo que distintos autores han venido denominando "clima psicológico y moral en el trabajo". Las variables a las que haremos referencia son: comunicación, cooperación, satisfacción en el trabajo y motivación, estilos de supervisión y actitudes y cultura. Todas ellas inciden de forma directa y continua en el comportamiento de los operadores en relación con su seguridad.

Comunicación: Las conductas de los individuos, bien coordinadas entre sí, son los primeros medios con que cuenta la organización para lograr sus objetivos, de ahí su importancia. Desde los clásicos trabajos de Bavelas en los años cincuenta sobre comunicación, han sido muchos los estudios realizados en el ámbito de las organizaciones en relación con el tema de la seguridad laboral (Cuny, 1969; Jablin, 1979; Grosjean y Lacoste, 1999).

Parece claro que para llevar a cabo cualquier tipo de orden o consigna, previamente hay que comprenderla, lo que implica compartir unos determinados códigos profesionales. En consecuencia, para crear un buen sistema de comunicación, habría que establecer entre las personas que en él participan, relaciones que impliquen una actitud favorable hacia los intercambios de información. No obstante, a veces, en las organizaciones actuales puede darse una sobrecarga de insumo informativo ante la que el individuo responde de forma ineficaz, generando graves disfuncionamientos en el sistema.

En los últimos años se subraya la necesidad de una ampliación de los códigos formales a todas las comunicaciones codificadas entre los obreros. No es deseable que una parte de éstas se abandonen al reino de lo informal, sino que, por el contrario, deben ser codificadas para que puedan constituir el objeto de un aprendizaje codificado, seguro y eficaz.

Cooperación y relaciones de grupo: Algunos estudios han mostrado la existencia de una estrecha relación entre ciertas normas, actitudes y condiciones de un grupo de trabajo y las tasas de accidentabilidad. En este sentido, los índices de accidentes parecen disminuir en la medida en que aumentan la cohesión y la integración en el grupo (Yoshimura, Sasou y Takano., 1994)).

Las relaciones con los compañeros (informales), según Pavard (1994), søn más importantes que las relaciones con los superiores (formales) en relación con la seguridad. En general, los sujetos poliaccidentados mantienen un "estatus" sociométrico marginal y malas relaciones profesionales. 
Motivación y satisfacción laboral: La mayoría de los autores que han trabajado sobre el tema coinciden en señalar que cualquier acción operativa en seguridad industrial que afecte al factor humano en relación con el grupo, sólo tendrá éxito si parte de la realidad que implica el conocimiento de las necesidades de todos los individuos que integran la organización (Studensky, 1975).

En el campo de la prevención de accidentes, la aplicación de modelos motivacionales es de gran importancia, ya que para potenciar comportamientos realmente seguros es necesario que los trabajadores, además de "poder" y "saber" ejecutar las tareas que se le asignan, "quieran" desarrollarlas de forma eficaz y segura; es decir, estén motivados para ello.

De otra parte, los estudios realizados sobre satisfacción en el trabajo en relación con accidentes no han aportado mucha luz. Sin embargo, el sentimiento personal de satisfacción o insatisfacción en el empleo se ha venido relacionando con tres fuentes principalmente: características específicas del puesto, grado de adaptación al lugar de trabajo y relaciones interpersonales entre compañeros. Parece lógico pensar que tales características estén relacionadas de algún modo con la seguridad en el trabajo. Así lo sugieren explícita o implícitamente los trabajos de Pestonje et al. (1977) y Allodi y Montgomery (1979).

Estilos de supervisión: La relevancia de esta variable deriva de la posición que ostentan determinadas personas en las organizaciones, tanto desde el punto de vista formal como informal, y de la influencia que como modelos tiene sobre el comportamiento seguro o inseguro de los trabajadores.

French y Raven, a finales de los años cincuenta, identificaron varias fuentes de poder para influir sobre los demás. De estas fuentes de influencia parece desprenderse, según las investigaciones llevadas a cabo por distintos autores (Tuttle et al., 1975), que las más importantes para generar comportamientos seguros son el poder de estímulo, el poder atribuido, el poder de competencia y el poder legítimo.

Otros autores estudian distintos aspectos relacionados con los estilos de liderazgo y sus repercusiones sobre la accidentabilidad. Así, en unos casos, se subraya la importancia del mando intermedio en la seguridad de la empresa; en otros, se interesan por la eficacia de los diversos estilos en las organizaciones o por las relaciones de contingencia; también se hace hincapié en la influencia del supervisor sobre los empleados y, finalmente, se estudian las posibles influencias del establecimiento de metas y de la supervisión sobre las conductas relacionadas con la seguridad de los trabajadores.

Actitudes: La prevención de riesgos exige un cambio de actitud y de cultura en las empresas. España se sitúa en el primer país de la Unión Europea en cuanto a 
indices de siniestralidad laboral. Además del incumplimiento de la Ley de Prevención de Riesgos Laborales por parte de las organizaciones empresariales y de los trabajadores, la falta de concienciación es el elemento clave y principal responsable del fenómeno de la accidentabilidad laboral.

Durante los últimos años la cifra de accidentes mortales ha sufrido un crecimiento sostenido. CCOO y ANEPA señalan que el principal causante de la alta siniestralidad es el escaso cumplimiento de la ley de Prevención de Riesgos Laborales, la falta de presupuestos destinados a la prevención, la escasez de personas con formación específica en la materia y, sobre todo, la escasa sensibilización de las empresas.

En un estudio elaborado por la consultora William $M$. Mercer sobre los servicios de prevención en 26 empresas de todos los sectores se pone de manifiesto la dificultad que tienen las compañias para valorar los riesgos y asignar un presupuesto a tal fin. De él se deduce que un $54 \%$ de las compañias analizadas no utilizan un presupuesto para el área de prevención de riesgos laborales, frente a un $46 \%$ que sí lo hace.

Esta actitud se deriva de la idea generalizada de que la seguridad es más un gasto que una inversión, pues aunque los grandes grupos empresariales desarrollan actividades preventivas desde hace ya algún tiempo, sigue siendo el sector de las PYMES, que es donde se producen la mayor parte de los accidentes, en el que se dan mayores resistencias. Los diferentes agentes implicados reclaman también un cambio en la actitud de la Administración, que debería convertirse en la principal promotora del cambio de la cultura de la prevención.

Parece que existe, en suma, una actitud de indiferencia generalizada hacia la prevención y hacia la seguridad laboral entre trabajadores, empresarios y Administración, según se desprende de los resultados de las encuestas y de las estadísticas que habrá que transformar mediante el desarrollo de actitudes positivas.

VARIABLES TÉCNICO-ORGANIZATIVAS:

Desde hace algunas décadas, una vez que adquirieron importancia los estudios ergonómicos, la investigación desplazó su atención desde los supuestos tradicionales de la "propensión" a la "influencia de las características de la organización; presiones de rol y demandas del trabajo o de la tarea" (Beehr y Newman, 1978), como potenciales estresores con incidencia directa sobre la seguridad, de tal forma que hoy contamos con excelentes métodos e inventarios para analizar las exigencias del comportamiento en los diversos puestos de trabajo, tales como el "Occupational Analysis Inventory" y el "Position analysis Questionaire". 
Puesto de trabajo: Los estudios realizados en torno a esta variable han sido numerosos, orientados fundamentalmente hacia las características de la tarea y cargas de trabajo (Wisner, 1974); ritmos de trabajo (Rasmussen, Duncan \& Leplat, 1987); y adaptación al trabajo (Laflamme, 1990). La relación de estas áreas con la accidentabilidad laboral parece estar fuera de toda discusión; sin embargo, aún no existen resultados definitivos, debido a dificultades metodológicas y de aplicación. Los avances, no obstante, que se produzcan en esta área, deben llegar de la perspectiva ergonómica (Özok y Salvendi, 1996).

En relación con este enfoque y con las características de la tarea, existen otras variables de gran relevancia para explicar el fenómeno de la accidentabilidad. ampliamente estudiadas y de gran tradición en la disciplina: condiciones ambientales, estado de la tecnología, rama de actividad, tamaño de la empresa, actividad y turnos de trabajo. No obstante, aunque parece obvia su importancia, no existen aún resultados definitivos, sobre todo en cuanto alas interacciones de estas variables con aquellas otras de carácter psicológico y social.

\section{VARIABLES PERSONALES DE MADUREZ:}

Bajo este rótulo incluimos las variables edad y experiencia en relación con la accidentabilidad laboral. Estas variables plantean problemas metodológicos si no se controlan de forma adecuada, ya que se da un solapamiento claro entre ambas. Esta observación cobra aún mayor sentido si partimos del supuesto real de que aquellos trabajadores con registros más elevados de accidentes, tienden a ser relevados del puesto o de la empresa; por tanto, cuanto más tiempo permanezca un empleado en el puesto de trabajo, tanto más probable es que sea más seleccionado con respecto a las conductas de seguridad. De ahí que una selección progresiva podría hacer aparecer una falsa relación negativa entre dichas variables.

Edad: El envejecimiento progresivo de la población, atribuido a las tasas de natalidad decrecientes, al incremento de la población de edad adulta y al incremento de la esperanza de vida, modificará el mercado laboral en los próximos años.

Según datos de la OCDE y para sus 24 países miembros (entre ellos España), la proporción de trabajadores con edades entre 45 y 64 años respecto a la población total de trabajadores (de 14 a 64 años) pasará del 32\% de 1980 al 41 '3\% esperado para el 2005; es decir, para esta fecha algo menos de la mitad de los trabajadores tendrá más de 45 años. En el caso de España se estima que en el año 2020 habrá 40'6 millones de habitantes, de los que, aproximadamente, el 16'5\% superará los 65 años. 
Se han realizado numerosos estudios sobre los trabajadores de edad avanzada; uno de ellos es el efectuàdo por el Finnish Institute of Occupational Health, publicado en la revista Hazards in the office, 1997 (6), 11. En él se afirma que es posible mejorar las capacidades individuales de los trabajadores a cualquier edad y se recomienda relacionar tres factores para poder desarrollar tales capacidades en los trabajadores mayores: el factor ergonómico y ambiental de la organización, la gestión del trabajo y el relativo a los factores individuales.

Experiencia: Tampoco esta variable es fácilmente aislable por lo que hasta el momento los estudios realizados no han aportado datos concluyentes. Parece obvio que la ejecución de cualquier trabajo exige poseer un conjunto de conocimientos necesarios para su correcta y eficaz ejecución, así como una preparación práctica que sólo se adquiere con la experiencia (Salthouse, 1990). Es lo que los anglosajones llaman el "saber cómo" conjugar ese conjunto de conocimientos teóricos y el conjunto de automatismos que permiten realizar una determinada tarea con un mínimo de esfuerzo y un máximo de eficacia.

La práctica se considera que mejora las aptitudes en un doble sentido: perfeccionamiento y compensación de deficiencias, por lo que, a primera vista, un alto grado de experiencia debería incidir sobre la disminución de los índices de accidentes.

En este sentido, podemos decir que, si bien la mayor parte de los estudios coinciden en valorar la experiencia como una variable relevante en la disminución de los accidentes laborales, también existen algunos autores que la valoran justo al contrario. Estas discrepancias creo que son explicables si tenemos en cuenta las interacciones existentes con otras variables muy difíciles de controlar. En todo caso, las estadísticas aportan datos irrefutables, pues se indica que cada año se incrementan los accidentes que se producen entre trabajadores con menos de un año de antigüedad en la empresa, entre los cuales se localiza casi la mitad del total de accidentes.

\section{ESTRATEGIAS ORIENTADAS A REDUCIR LA ACCIDENTABUIDAD LABORAL}

Al inicio del capítulo hablamos de la importancia de las medidas de ingeniería en el diseño, ubicación, mantenimiento y protección de los equipos, cuya incidencia en la reducción de accidentes o en el incremento de la seguridad está fuera de toda discusión. Sin embargo, también dijimos que tales medídas eran del todo insuficientes dado que las máquinas y los equipamientos por sí soløs no pueden realizar los procesos de transformación que exigen los sistemas de pro:ducción, sino que éstos han de operar en conjunción con el componente huma- 
no. En consecuencia, nosotros nos centraremos en aquellas estrategias que, desde el punto de vista psicológico, pueden ayudar a potenciar la seguridad en el contexto del trabajo.

El punto de partida será lograr un ajuste satisfactorio entre el trabajador y su lugar o puesto de trabajo. Por tanto, una primera estrategia basada en las diferencias individuales será la selección del personal, mediante la cual podremos identificar las demandas del puesto de trabajo y las características o requisitos deseables entre quienes opten a desempeñarlo.

Esta aproximación, basada en la investigación de las diferencias como predictoras de accidentes, ha seguido dos tendencia, una basada en el grupo (edad, sexo, estado civil ...), como predictor y otra basada en el individuo. La primera de ellas ha resultado ser un potente predictor de accidentes. De hecho, las compañías de seguros se basan en tales criterios, entre otros, a la hora de establecer las primas de sus asegurados. La segunda de esas tendencias se basa en la identificación de las características individuales de los candidatos sobre la que los psicólogos han investigado mucho y durante largo tiempo, si bien los resultados no han sido prometedores. No obstante, algunas variables parecen ser potentes al predecir en ciertas situaciones o puestos de trabajo: la capacidad de percepción en las conductas relacionadas con la conducción (Mihal y Barret, 1976; Williams, 1977); algunas variables de personalidad como agresividad o ansiedad en relación con las conductas inseguras (Wilson y Greensmith, 1983). De todos modos, como ya se puso de manifiesto en el estudio de las variables psicológicas, no parecen ser predictores consistentes, en general, de las conductas seguras.

Una segunda estrategia que podemos aplicar para reducir la accidentabilidad laboral está basada en la psicología de la ingeniería, desde la que se intentará modificar el puesto de trabajo y adecuarlo a las características del operador: rediseño del equipo, herramientas y maquinaria, reubicación y protecciones orientadas a incrementar las condiciones de seguridad de los trabajadores que han de operar en ellas. El psicólogo habrá de centrar sus esfuerzos, en este caso, en identificar los desajustes que se producen en el trinomio hombre-máquina-entorno con el fin de crear un sistema seguro en donde el trabajador pueda ejecutar su actividad con el mínimo riesgo (Adams, 1972; McCormick y Sanders, 1982).

El diseño de contextos de trabajo seguros ha sido uno de los objetivos más antiguos y prioritarios que ha tenido la Psicología de la ingeniería, estando fuera de toda discusión el alto grado de eficacia conseguido en el campo de la seguridad laboral.

En tercer lugar, suelen aplicarse con un alto nivel de efectividad las estrategias de adiestramiento del personal, cuyo objetivo principal es mejorar las habilidades de los trabajadores para que puedan afrontar con mayor competencia y seguridad 
las actividades que desarrollan. No obstante, para que los programas de adiestramiento tengan éxito han de ser asumidos y apoyados por los propios trabajadores y deben representar para ellos una verdadera fuente de motivación, lo que se consigue creando un clima favorable hacia la seguridad en la organización en su conjunto (Zohar, 1980), en donde las actitudes de los directivos y mandos intermedios son de considerable importancia. De otro lado, no hay que olvidar que las conductas seguras requieren una atención sistemática y continuada, no basta con la implantación de programas concretos.

En los programas de entrenamiento suelen utilizarse múltiples técnicas, entre las que cabe destacar todas aquellas relacionadas con la estimulación de la motivación de los individuos hacia la seguridad (programa de refuerzos), con la modificación y cambio de actitudes (técnicas de sensibilización) hacia conductas seguras y técnicas de pedagogía activa orientadas específicamente al logro de una mayor habilidad y competencia profesional en las distintas actividades que realizan los trabajadores (Reber, Wallin y Chokar, 1984). A través del desarrollo de estos programas y de estas técnicas podemos realizar un amplio abanico de actividades orientadas a la prevención, tales como:

- Sensibilización para una cultura de la prevención.

- Promoción y apoyo de la actividad preventiva en el trabajo.

- Programas de formación orientados a la prevención.

- Fomento mediante incentivos de la actuación preventiva.

- Refuerzo de las actividades de investigación, análisis y estudio.

- Acciones legislativas y acciones complementarias para la aplicación de la normativa de prevención de riesgos laborales.

- Refuerzo de la eficacia de las actuaciones en materia de vigilancia y de control.

- Coordinación y cooperación institucional entre los distintos organismos de las Administraciones Públicas y entre éstos y las Fundaciones y Agentés sociales y económicos implicados en los temas de prevención de riesgos.

Por último, el denominador común de las estrategias de adiestramiento es su orientación a potenciar las conductas de seguridad en un sentido positivo: Raramente se diseñan programas que se entren en conductas inseguras, o en la violación de las normas de seguridad.

Estas tres aproximaciones a las que nos hemos referido no sólo no son excluyentes, sino que deben darse de forma conjunta, pues cada método se aproxima a la seguridad desde una perspectiva distinta, pero serán los tres de forma conjunta los que provean de una seguridad efectiva a los trabajadores en sus distintos eanopos de actividad. 


\section{BIBLIOGRAFLA}

- ALONSO, F., et al. (1979), "Validez de los exámenes para la prevención de accidentes de trabajo". Revista de Psicología General y Aplicada, 159, pp. 711-720.

- AMAIBERTI, R.,(1996). La conduite de systèmes à risques. París. PUF.

- AMALBERTI, R., \& MOSNERON-DUPIN, F. (eds.) (1997). Facteurs humains et fiabilité. Toulouse: Octarés.

- BRAVERMAN, H. (1975) Trabajo y capital monopolista. La degradación del trabajo en el siglo XX. México: Nuestro Tiempo.

- BÉGUIN, P., \& WEILL-FASSINA, A. (eds.) (1997). La simulation en ergonomie: connaire, agir et interagir. Toulouse: Octarès.

- BIRD, F. E. (1974). Management guide to loss control. Sta. Mónica, CA: Institute Press.

- BREHMER, B., \& ALLARD, R. (1991). Dynamic decision making: the effects of task complexity and feedback delay. In J. Rasmussen, B. Brehmer, \& J. Leplat (eds.). Distributed decision making: cognitive models for coopeerative work (pp. 319-334). Chichester, UK: Wiley \& Sòns Ltd.

- CABERNI, J-P. (1998). L'ethique dans les sciences du comportament. París: PUF, coll. Que sais-je?.

- CACCIABUE. P.C. (1998). Modelling and simulation of human behavior in sytem control. Berlin: Springer Verlag.

- CARLOSSON, J. (1980). "Accident prevention planning, II. Suggestions for improvements". Elmia-Arbetsmiltjo, 17-25.

- CARVEY, D. V. y NIBLER, R. G. (1977). "Biorhythmic cycles and the incidence of industrial accidents". Personnel Psychology, 30, 3, 447.454.

- CASTELFRANCHI, C. (1998). Modelling social action for agents. Artificial Intelligency, 103, 157-182.

- CASTEllaN Jr, N (ed.) (1993). Individual and group decesión making-current Issues. Hillsdale, N: Lawrence Erlbaum Associates.

- CHARNESS, N.,1985. Aging and human performance. Wiley\&Sons. Univ. of Waterloo, Canada.

- CLOT, Y.,1999. La fonction psychologique du travail. Contribution à une analyse psychologique de l'action. París. PUF.

- CUNY, X. (1969) "Semiologie et études ergonomiques des communications de travail". Le Travail Humain, 32, 3-4, 177-198.

- DERRIENNIC, F., TOURANCHET, A., \& VOLKOFF, S. (1992). Enquête ESTEV: un instrument d'estude des relations entre âge, santé et travail. Arcbives des Maladies Professionelles, 53, 79-89.

- DE REAMER, R. (1980). Modern safety and health technology. Nuva York: Wiley.

- DODIER, N. (1995). Les hommes et les machines. La conscience collective dans les societés technicisées. París: Editions Metailié.

- DRISKELL, J \& SALAS, E. (1992). Collective behaviour and team performance. Human Factors, 34, 227-288. 
- FEYER, A. \& WILLIAMSON, A. (eds). (1998). Ocupational injury: Risk, prevention, and intervention. London: Taylor \& Francis.

- FLOODY, D. R. (1981). "Further systematic research with byorhythms" Journal of Applied Psychology, 66, 520-521.

- GLENDON, A. \& MCKENNA, E. (1995). Human safety and risk management, London: Chapman \& Hall.

- GROSJEAN, M \& LACOSTE, M., 1999. Communication et intelligence collective. París. PUF.

- GaNASCIA J., (ed.) (1998). Sécurité et cognition. París. Hermès.

- HALE, M \& HALE, A.,1986. A revew of literature relative to the accident experience of young workers and the relation between accidents and age. Birmingham, UK: Health and Safety Technology and Management Hastam Ltd.

- HEBERT, F., 1996. Les indicateurs de lésions professionnelles en santé et sécurité du travail: analyse par secteur d'activité économique en 1991. Montreal, Canada: Institut de Recherche en Santé et en Sécurité du Travail.

- HELM, J. D. (1978). "Personality predictors of accident tendences in construction workers". Disertation abstracts Interncional, 38, 2, 225-232.

- GALEGHER, J., KRAUT, R., \& EGIDO, C. (eds) (1990). Intellectual teamwork-social and technological foundations of cooperative work. Hillsdale, Nj: Erlbaum.

- HOC, J. M. (1998). How can we evaluate the quality of human-machine cooperation? In F. Darses \& P. Zaraté (Eds.), COOP 98. Third International Conference on the Design of Cooperative sytems (pp. 121-130). Le Chesnay, F: INRIA.

- HOLLNAGEL, E. (ed.). (1993). Human reliability analyssis, context and control. London: Academic Press.

- IZARD, C.E. (1991). The psychology of emotions. New York, PA: Plenum Press.

- KEYSER DE V. (1990). Fiabilité humaine et la gestion du temps dans les systémes complexes. In J. Leplat \& G. De Terssac (eds.), Les facteurs humains de la fiabilité dans les systèmes complexes (pp. 85-110). Toulouse: Octarés.

- KATZ, D. y KAHN, R. L. (1978). The Social Psychology of Organizations Nueva York: Wiley ( $2^{2}$ ed. Revisada).

- KLETZ, T. (1994). Learning from accidents. Oxford, UK: Butterworth Heinemann.

- KOUABENAN, D.,1999. L'explication naïve de l'accident: Contribution psychosociale á l'étude et à la prévention des accidents. París. PUF.

- LA BENZ, P.; Cohen, A. y Pearson, B. (1997) "A noise and hearing survey of hearthmoving equipment operators". American Industrial Hygiene Association Jornal, 28, 117-128

- LAFLAMME, L., 1990. A better understanding of occupationalaccident genesis to improve safety in the workplace. Journal of Occupational Accidents, 12, 155-165.

- LANT, T. (1989). Simulation games: A researh method for studying organizational behavior. New York, PA: New York University.

- LAVILlE, A. (1976). L'ergonomie. París: PUF.

- LAVILlE, A., (1989). Vieillissement et travail. Le Travail Hümaine, 52, p. 3-20.

- LEPLAT, J \& DE TERSSAC, G (eds) (1990). Les facteurs humains de la fiabilite. Toúloùse: Octarès. 
la Prevención de Riesgos y la accidentabilidad laboral. Aportaciones deside la Psicologia Social 101

- LEPLAT, J. (1991). Activités collectives et nouvelles technologies. Revue Internationale de Psycbologie Sociale, 4, 335-356.

- LEPLAT, J., 1997. Regards sur l'activité en situation du travail. París. PUF.

- LEPLAT, J. y CUNY, X. (1979). L'accident du travail. París: PUF (2a de.)

- LEVINE, J. et al. (1973). The effect of alcohol on human performance: a classification and integration of research finding. Washington: American Institute for Research.

- LEVENSTEIN, C., \& WOODING, J. (Eds). (1997). Work, and envioronment. New York: The Guilford Press.

- LOPEZ MENA, L. (1978). "Evaluación de las actitudes de los trabajadores hacia la seguridad industrial". XIX Congreso Internacional de Psicología Aplicada, Munich.

- MHAL, W. L. y BARRET, G. W. (1976). "Individual differences inperceptual information processing and their rellation to automobile involvement". Journal of Applied Psychology, 61, 229-233.

- MOYEN, D.; QUINOT, E. y HEIMFERT, M. (1980) "Exploitations d'analyses d'accidents du travail a des fins de prevention. Essay methodologique". París: INRS, rep. 23.

- McCOMICK, E. J. (1980-82). Human factors inengineering and design. Nueva York: McGraw-Hill

- ÖZOK, A.F \& SALVENDY, G (Eds.) (1996). Advances in applied ergonomics. Istanbul, Turkey: Usa Publising Corporation-West Lafayette.

- PAVARD, B. (Ed.) (1994). Systèmes coopératifs: de la modélisation à la conception. Toulouse: Octarés.

- PEIRO, J. M. (1984) "Psicología organizacional. Nuevas aproximaciones al estudio de la conducta organizacional". En J. Mayor (de.) Actividad Humana y procesos Cognitivos. Madrid: Alhambra.

- PERSINGER, M. A. et al. (1978). "No evidence for realtionshipbetween biorhythms and industreial accidents". Perceptual and Motor Skills, 46, 423-426.

- PESTONJEE, D. M. et al. (1977). "Employees morale and industrial accidents". Indian Journal of Soc. Work., 38, 1, 70-85.

- RABARDEL, P. (1995). Les activités avec instruments. París: Armand Colin.

- RASMUSSEN, J, BREHMER, B \& LEPLAT, J (eds.) (1991). Distributed decission making: Cognitive models for cooperative work. Chichester, UK: John Wiley \& Sons Ltd.

- RASMUSSEN, J. DUNCAN, K. \& LEPLAT, J. (eds.) New tecnology and human error. Chichester, UK: Wiley.

- REASON, J. (1990). Human error. Cambridge: Cambridge University Press.

- REASON, J. (1995). A systems approach to organizational error. Ergonomics, 38, 1708-1721.

- ROGAISKI J. (1994). Formation aux activités collectives. Le Travail Humain, 57, 425-443.

- ROMAN, W. Et al. (1973). "Effects of goal setting and supervision on worker behavioir in an industrial situation". Journal of Applied Psychology, 58, 3, 302-307.

- SABAH, G., VIVIER, J., VILNAT, A., PIERREL, J.-M., ROMARY, L., \& NICOLlE, A. (1997). Machine, langage et dialogue. París: L'Harmattan, 342 p.

- SALTHOUSE, T. A. (1990a). Influence of experience on age differences in cognitive fonctionning. Human Factors, 32, 15, 551-569. 
- SALTHOUSE, T. A. (1990b). Cognitive competence and expertise in aging. In J. E. Birren \& K. W. Schaie (Eds (1990b). Handbook of the psychology of aging (pp. 310-319). San Diego, CA: Academic Press ( $3^{a}$ ed.).

- SEBEK, 1. y KONRAD, E. (1972). Analysis of accidents or working teams. Univ. Ljubljana Press.

- SIGUÁN, M. (1981). "El factor humano en los accidentes". Mapfre Seguridad, 2, 33-41.

- SPERANDIO, J. (1980). La Psychologie en Ergonimie. París, PUF.

- STUDENSKY, R. (1975). "Application of techniques of aspiration level measurement to research on the effect of motivtion on efficient action". Psychol. Wychowawcz., 18, 4, 550-558.

- TEIGER, C. (1989). Le vieillissement différentiel dans et par le travail. Un vieux probleme dans un contexte récent. Le Travail Humain, 52, 21-56.

- TERSSAC de, G. (1992). Autonomie dans le travail. Sèrie "Sociologie d'Aujord'hui". París: PUF.

- TOPF, M. (1985) "Personal and environmental predictors of pacient disturbance due to haspital noice". Journal of Applied Psychology, 70, 22-28

- TRINQUET, P., 1996. Maîtriser les risques du travail. París. PUF.

- VICKROY, S. C. et al. (1982). "Effects of temperature, clothing and task complexity on task performance and satisfaction. Journal of applied Psychology, 67, 97-102.

- VOLKOFF, S., (1989). Le travail aprés 50 ans: quelques chiffres et plusieurs inquiétudes. Le Travail Humain, 52, 97-116.

- VOLKOFF, S., LAVILLE, A., \& MAILlARD, M.-C. (1992). Age et travail: contraintes, sélection et difficultés chez les 40-50 ans. Une analyse de l'enquête ESTEV. Travail et Emploi, $4,20-33$.

- VOLKOFF, S., \& MOLINIÉ, A. A.-F. (1995). Eléments pour une démographie du travail. In J. C. Marquié, D. Paumés, \& S. Volkoff (Eds.), Le travail au fil de l'âge (pp. 99-129). Toulouse, Octarés.

- WAERN, Y. (Eds). (1998). Co-oporative process managemet: cognition and information technology. London: Taylor \& Francis.

- WILSON, T. y GREENSMITH, J. (1983). "Multivariate analysis of the relationship between drivometer variables an drivers' accident, sex and exposure status". Human Factors, 25, 303-312.

- WILLIAMS, T.A. et al. (1982). "Stress, alcoholism and personality ". Humans Relations, 35, $6,491-510$

- WISNER, A. (1974). "Contenu des taches et chargede travail". Socialigie du travail. 4, 339357.

- MIDDLETON, D \& ENGSTRÖM, Y (eds) (1996), Communication and cognition at work. New York, Cambridge University Press.

- YOSHIMURA, S., SASOU, K., \& TAKANO, K. (1994). Team behavior simulation model: Propasal of the conception. Journal of Scientific E Industrial Research, 53,:574-578: 